\title{
Chronic ethanol consumption increases the levels of chemerin in the serum and adipose tissue of humans and rats
}

\author{
Rui-zhen REN ${ }^{1,2,3, \#, ~ X u ~ Z H A N G}{ }^{1,2, \#}$, Jin XU ${ }^{1,2}$, Hai-qing ZHANG ${ }^{1,2}$, Chun-xiao $\mathrm{YU}^{1,2}$, Ming-feng $\mathrm{CAO}^{1,2}$, Ling GAO ${ }^{4}$, Qing-bo
} GUAN $^{1,2, *}$, Jia-jun $\mathrm{ZHAO}^{1,2, *}$

${ }^{1}$ Department of Endocrinology, Provincial Hospital Affiliated to Shandong University, Ji'nan 250021, China; ${ }^{2}$ Institute of Endocrinology, Shandong Academy of Clinical Medicine, Ji'nan 250021, China; ${ }^{3}$ Department of Endocrinology, Yantai Yuhuangding Hospital Affiliated to Qingdao University, Yantai 264000, China; ${ }^{4}$ Central Laboratory, Provincial Hospital Affiliated to Shandong University, Ji'nan 250021, China

Aim: Chemerin is a new adipokine involved in adipogenesis and insulin resistance. Since ethanol affects the insulin sensitivity that is closely associated with adipokines. The aim of this study was to investigate the effects of ethanol on chemerin in humans and rats. Methods: In the human study, 148 men who consumed alcohol for more than 3 years and 55 men who abstained from alcohol were included. Based on ethanol consumption per day, the drinkers were classified into 3 groups: low-dose ( $<15$ g/d), middle-dose (15$47.9 \mathrm{~g} / \mathrm{d})$ and high-dose ( $\geq 48 \mathrm{~g} / \mathrm{d})$. Anthropometric measurements and serum parameters were collected. In the rat study, $27 \mathrm{male}$ Wistar rats were randomly divided into 4 groups administered water or ethanol $\left(0.5,2.5\right.$, or $\left.5 \mathrm{~g}^{\prime} \mathrm{kg}^{-1} \cdot \mathrm{d}^{-1}\right)$ for $22 \mathrm{weeks}$. The chemerin levels in the sera, visceral adipose tissue (VAT) and liver were measured using ELISA.

Results: In the high-dose group of humans and middle- and high-dose groups of rats, chronic ethanol consumption significantly increased the serum chemerin level. Both the middle- and high-dose ethanol significantly increased the chemerin level in the VAT of rats. In humans, triglyceride, fasting glucose, insulin and HOMA-IR were independently associated with chemerin. In rats, the serum chemerin level was positively correlated with chemerin in the VAT after adjustments for the liver chemerin $(r=+0.768)$. High-dose ethanol significantly increased the body fat in humans and the VAT in rats.

Conclusion: Chronic ethanol consumption dose-dependently increases the chemerin levels in the serum and VAT. The serum chemerin level is associated with metabolic parameters in humans. The increased serum chemerin level is mainly attributed to an elevation of chemerin in the VAT after the ethanol treatment.

Keywords: ethanol; adipokine; chemerin; insulin resistance; triglyceride; adipose tissue

Acta Pharmacologica Sinica (2012) 33: 652-659; doi: 10.1038/aps.2012.11; published online 26 Mar 2012

\section{Introduction}

Adipose tissue is classically considered a tissue that stores excess energy and provides insulation for the body; however, it is now considered to be an endocrine organ. The adipose tissue secretes multiple metabolic proteins known as adipokines, and some of these play important roles in glucose and lipid metabolism, insulin resistance, obesity and type 2 diabetes $^{[1]}$.

Chemerin, a newly found adipokine, is secreted as an

\footnotetext{
\#These authors contributed equally to this work.

* To whom correspondence should be addressed.

E-mail jjzhao@medmail.com.cn (Jia-jun ZHAO); guanqingbo@medmail.com.cn (Qing-bo GUAN)

Received 2011-11-16 Accepted 2012-02-01
}

$18-\mathrm{kDa}$ inactive proprotein named prochemerin and is converted into the $16-\mathrm{kDa}$ active chemerin by a serine protease cleavage of the C-terminal portion of the protein ${ }^{[2]}$. It is most highly expressed in the white adipose tissue and liver, which are followed by the lung, kidney, pituitary, placenta and ovary ${ }^{[3-5]}$. Chemerin is a novel chemoattractant protein that plays roles in adaptive and innate immunity ${ }^{[2,5]}$. Recent research found that chemerin participated in the regulation of adipocyte differentiation ${ }^{[3,4]}$ and had effects on insulin sensitivity. Until now, the majority of studies have demonstrated that chemerin induces insulin resistance in the adipose tissue and skeletal muscle $\mathrm{e}^{[6-8]}$.

Ethanol consumption is a lifestyle factor and is relevant to type 2 diabetes. Moderate alcohol consumption and a high amount of alcohol intake produced different effects on lipid 
metabolism and insulin sensitivity ${ }^{[9,10]}$. Until now, few studies have focused on the effects of ethanol on adipokines. Our previous study demonstrated that ethanol consumption elevated the leptin, resistin and visfatin levels and decreased the adiponectin concentrations in both the sera and visceral adipose tissues (VAT) of rats ${ }^{[11]}$. Unlike the other adipokines, chemerin was highly expressed in the adipose and liver tissues. Ethanol affects liver function, lipid metabolism and insulin sensitivity. Until now, there was no study on the correlations between chemerin and ethanol. Therefore, this study aimed to observe the effects of a long-term intake of different doses of ethanol on chemerin in humans and rats and to evaluate the relationship of chemerin with metabolic parameters in humans.

\section{Materials and methods \\ Study 1: Human study \\ Subjects}

Data were obtained from an epidemiological investigation of type 2 diabetes in the Shandong Provincial Hospital in China from September 2009 to June 2010. All of the participants were from two central communities (Shungen and Yangguang in Ji'nan, China), and were male who were 22-75 years of age and had been living here for more than 5 years. A total of 1502 men participated in the investigation. According to the study criteria, 148 men who consumed alcohol and 55 men who abstained from alcohol were included in the study.

The exclusion criteria were dependent on a self-reported history and the data from our measurements. The body mass indexes (BMI) of all of the subjects were less than 25 $\mathrm{kg} / \mathrm{m}^{2}$. Subjects were excluded for diabetes, any acute or chronic inflammatory diseases, hepatic diseases, liver function disorders, hypertension, cardiovascular or cerebrovascular diseases, renal diseases or thyroid diseases. None of the subjects were taking any medication that was known to influence glucose and lipid metabolism, blood pressure or weight. Each participant completed a self-administered questionnaire that included demographic characteristics, an ethanol and smoking history, a general health status and current medications. This study was approved by the Ethics Committee of the Shandong Provincial Hospital. The purposes and procedures of the study were explained to the participants prior to the questionnaire and fasting blood draw. Informed consents were obtained from all of the participants.

The ethanol intake was assessed by the frequency and amount of intake (a frequency of less than once per week was excluded). The average alcohol intake per day was calculated by the average quantity per occasion multiplied by the drinking frequency per week divided by seven. The drinkers were classified into three groups: a low-dose group (group L; ethanol consumption $<15 \mathrm{~g} / \mathrm{d}$ ), a middle-dose group (group $\mathrm{M}$; ethanol consumption 15-47.9 g/d), and a high-dose group (group $\mathrm{H}$; ethanol consumption $\geq 48 \mathrm{~g} / \mathrm{d})^{[12,13]}$. The length of time that each participant had been drinking was more than three years, and there was no difference among the three groups.

\section{Anthropometric measurements}

A complete physical examination was conducted on each individual under the condition of an empty bladder and stomach. The values of height and weight were taken with the subjects dressed in light clothing and without shoes. The height was determined to the nearest $1 \mathrm{~cm}$, and the weight was determined to the nearest $0.5 \mathrm{~kg}$. The BMI was calculated as the weight in kilograms divided by the height squared in meters. The waist circumference was measured at the midpoint between the lower costal margin and the iliac crest. The hip girth was measured as the horizontal circumference at the broadest part of the hip. The waist-to-hip ratio (WHR) was calculated as the waist circumference $(\mathrm{cm})$ divided by hip circumference $(\mathrm{cm})$. The percentage of body fat was assessed using the InBody720 body composition analyzer (Biospace, Seoul, Korea) and an established protocol.

\section{Laboratory measurements}

Blood samples were collected after an overnight fast. The serum samples were separated and immediately stored at $-80^{\circ} \mathrm{C}$. The fasting plasma glucose (FPG) concentration was determined by the glucose oxidase method. The fasting serum insulin (FINS) was measured using a radioimmunoassay kit (Northern Bioengineering Institute, Beijing, China). The plasma concentrations of total cholesterol (TC), triglyceride (TG), low-density lipoprotein cholesterol (LDL-C), and highdensity lipoprotein cholesterol (HDL-C) were measured using routine enzymatic methods with an Olympus 5400 Analyzer. The insulin sensitivity was estimated using the homeostasis model assessment for insulin resistance (HOMA-IR), which was calculated as fasting insulin (mU/L) multiplied by fasting glucose $(\mathrm{mmol} / \mathrm{L})$ divided by $22.5^{[14]}$.

\section{Study 2: Animal study}

\section{Animal protocols and housing}

All animal protocols and procedures were approved by the Shandong University Institutional Animal Care and Use Committee (Ji'nan, China). Twenty-seven male Wistar rats (weights of 200-240 g; 8 weeks old) were purchased from the Experimental Animal Center, Shandong University, China. All rats were kept on a 12-h light and 12-h dark cycle in individual cages and had free access to water and normal chow. The rats were divided into four groups and given the following different treatments: control group (group C; distilled water at $5.0 \mathrm{~g} \cdot \mathrm{kg}^{-1} \cdot \mathrm{d}^{-1}$ ), low-dose group (group L; ethanol at $0.5 \mathrm{~g} \cdot \mathrm{kg}^{-1} \cdot \mathrm{d}^{-1}$ ), middle-dose group (group $\mathrm{M}$; ethanol at $2.5 \mathrm{~g} \cdot \mathrm{kg}^{-1} \cdot \mathrm{d}^{-1}$ ), and high-dose group (group H; ethanol at 5.0 $\left.\mathrm{g} \cdot \mathrm{kg}^{-1} \cdot \mathrm{d}^{-1}\right)$. Distilled water or edible ethanol (50\% v/v; Ji'nan Baotu Spring Distillery, Shandong, China) was given by a gastric tube every morning for 22 weeks. Using a body surface area conversion ratio between rats and humans, the ethanol dosages of $0.5,2.5$, and $5 \mathrm{~g} \cdot \mathrm{kg}^{-1} \cdot \mathrm{d}^{-1}$ for the rats were equivalent to the dosages of 5.2, 27.3, and $53.95 \mathrm{~g} / \mathrm{d}$ for a man weighing 65 kilograms, respectively. These three increasing ethanol treatments in rats were equal to the low-, middle-, and highdose groups of humans. 


\section{Blood and tissue collection}

Blood samples from all of the rats were obtained from the inferior vena cava after anesthetization by an intraperitoneal injection of sodium pentobarbital $(0.1 \mathrm{~mL} / 100 \mathrm{~g}$ body weight after an overnight fast. The serum samples were separated after centrifugation and immediately stored at $-80^{\circ} \mathrm{C}$ for subsequent analyses. The epididymal and perirenal fat pads and livers were removed and rapidly frozen in liquid nitrogen for adipokine measurements.

\section{Laboratory measurements}

The FPG, FINS, and HOMA-IR were determined and calculated as described above. Samples of the liver and adipose tissues $(200 \mathrm{mg}$ ) were excised from the frozen specimens while on ice. The excised tissues were added to $200 \mu \mathrm{L}$ of phosphate-buffered saline (PBS) ( $\mathrm{pH} 7.4$ ) and homogenized using a VCX450 ultrasonic cell disruption system (Xinzhi Biotechnology Co, Ltd, Ningbo, China; homogenization parameters: time $=30 \mathrm{~s}$, temperature $=4^{\circ} \mathrm{C}$, pulse on $=3 \mathrm{~s}$, pulse off $=5 \mathrm{~s}$ and amplifier $=28 \%-30 \%$ ). The tissue homogenates were frozen overnight at $-80^{\circ} \mathrm{C}$ and thawed on ice the following day. After two freeze-thaw cycles, the homogenates were centrifuged with a high-speed refrigerated centrifuge (Beckman, Indianapolis, USA; Parameters: revolution $=15000$ rounds per minute, time $=30 \mathrm{~min}$ and temperature $=4^{\circ} \mathrm{C}$ ). The middle layer of the adipose tissue homogenate and the supernatant of the liver homogenate were isolated and stored at $-80^{\circ} \mathrm{C}$ to determine the total protein level and chemerin concentration. The total protein concentrations of the liver and adipose tissues were measured with a protein assay kit (BCA; Bio-Rad, Hercules, CA, USA).

\section{Enzyme-linked immunosorbent assay (ELISA)}

The chemerin levels of humans and rats were determined by commercially available ELISA kits according to the manufacturer's instructions (Uscn Life Science \& Technology Co, Ltd, Wuhan, China). The concentrations in the sera of humans and rats were directly determined without dilution. The isolated protein lysates were diluted to determine the chemerin levels because the isolated protein concentration of each liver and adipose tissue was different. Equal concentrations of isolated protein from the liver and VAT were taken to determine the chemerin content per $100 \mathrm{mg}$ of protein.

\section{Statistical analysis}

Before proceeding with the statistical analysis, all of the parameters were tested for a normal distribution using the Kolmogorov-Smirnov test. Parameters that were not normally distributed were transformed to approximate a normal distribution. Comparisons between different groups of normally distributed parameters were made using an ANOVA, which was followed by a least-significant difference (LSD) post hoc test, and the parameters with skewed variables were tested by a Mann-Whitney U test. An analysis of covariance was carried out to compare the differences among the four groups of humans after adjustments for age. The descriptive data are presented as the mean \pm standard deviation (SD) and medians (interquartile range). Correlation analyses were performed using Pearson's test. A multiple linear regression analysis was performed to identify independent factors affecting the chemerin levels. Statistical analyses were performed using SPSS (Statistical Package for Social Sciences) 12.0 for Windows (SPSS, Chicago, IL, USA). Statistical significance was defined as $P<0.05$.

\section{Results}

Anthropometric and metabolic characteristics of the human participants

Anthropometric and metabolic characteristics of the subgroups studied are presented in Table 1. Lipid components, including the HDL-C, TC, and TG levels, increased with an elevated ethanol intake. Likewise, anthropometric parameters, including the WHR, body fat, waist-hip ratio of body fat, and percentage of body fat, were also elevated in group $\mathrm{H}$. The FPG, FINS, and HOMA-IR in group $\mathrm{H}$ were also significantly elevated compared with group $\mathrm{C}$. Because the average age of group $\mathrm{H}$ was significantly higher than the average ages of group C, group L, and group M, we further adjusted for age among the groups. Only the body fat, percentage of body fat, waist-hip ratio of body fat, TG, FPG, FINS, and HOMA-IR in group $\mathrm{H}$ were significantly elevated compared with group $\mathrm{C}$ after the adjustment for age (Table 2).

\section{Chronic ethanol consumption increased serum chemerin in humans}

Chronic ethanol consumption caused a dose-dependent increase of chemerin in human sera. The chemerin levels in group $\mathrm{L}$, group $\mathrm{M}$, and group $\mathrm{H}$ increased by $9.75 \%(P=0.265)$, $13.84 \%(P=0.094)$, and $40.83 \%(P<0.001)$, respectively, compared to group $C$ (Table 1). Furthermore, even after the adjustment for age, chemerin remained significantly different among the groups (Table 2).

\section{Serum chemerin levels are associated with TG and HOMA-IR in humans}

A Pearson's correlation analysis was performed between the serum chemerin concentrations and the clinical characteristics (Table 3). The results showed that the chemerin levels positively correlated with the BMI, body fat, TG, FPG, FINS, and HOMA-IR and negatively correlated with the HDL-C. To study the factors that influenced the circulating chemerin, we performed a multivariate linear regression analysis using chemerin as the dependent variable (Table 4). The results revealed that the TG, FPG, FINS, and HOMA-IR were independently associated with the chemerin concentrations.

\section{Characterization of the rats}

The FINS levels and HOMA-IR of rats increased in the ethanol-treated groups compared with the controls. The rats in the four groups had similar body weights (BWs) at baseline. However, the BWs of group $\mathrm{H}$ decreased and the ratio of the epididymal adipose tissue weight to the $\mathrm{BW}$ of group $\mathrm{H}$ 
Table 1. Clinical and laboratory characteristics of different groups in human. Log10 transformed FINS, FPG, HOMA-IR, and TG were used for statistical analysis. Normally distributed data are mean \pm SD and analyzed by ANOVA. Nonnormally distributed parameters are median (interquartile range) and analyzed by Mann-Whitney U test. Group C, control group; group L, low-dose group (ethanol <15 g/d); group M, middle-dose group (ethanol $15-47.9$ $\mathrm{g} / \mathrm{d}$ ); group $\mathrm{H}$, high-dose group (ethanol $\geq 48 \mathrm{~g} / \mathrm{d}$ ); BMI, body mass index; WHR, waist-hip ratio; WHR of body fat (BF), ratio of waist fat to hip fat; PBF, percentage of body fat; TC, total cholesterol; HDL-C, high-density lipoprotein cholesterol; LDL-C, low-density lipoprotein cholesterol; TG, triglycerides; FPG, fasting plasma glucose concentration; FINS, fasting insulin concentration; HOMA-IR, homeostatic model assessment of insulin resistance. ${ }^{\mathrm{b}} P<0.05$, ${ }^{\mathrm{c}} P<0.01$ vs group C. ${ }^{\mathrm{e}} \mathrm{P}<0.05,{ }^{\mathrm{f}} \mathrm{P}<0.01$ vs group L. ${ }^{\mathrm{h}} \mathrm{P}<0.05,{ }^{\mathrm{i}} \mathrm{P}<0.01$ vs group $\mathrm{M}$.

\begin{tabular}{|c|c|c|c|c|}
\hline Characteristics & $\begin{array}{c}\text { Group C } \\
n=55\end{array}$ & $\begin{array}{c}\text { Group L } \\
n=54\end{array}$ & $\begin{array}{c}\text { Group M } \\
n=54\end{array}$ & $\begin{array}{c}\text { Group H } \\
n=40\end{array}$ \\
\hline Age (year) & $42.96 \pm 15.16$ & $42.61 \pm 14.25$ & $43.89 \pm 15.27$ & $51.08 \pm 12.30^{\mathrm{cfh}}$ \\
\hline $\mathrm{BMI}\left(\mathrm{kg} / \mathrm{m}^{2}\right)$ & 22.15 (20.79-24.10) & $22.64(21.13-24.23)$ & $22.47(21.59-23.65)$ & $23.17(20.99-23.84)$ \\
\hline WHR & $0.86(0.84-0.89)$ & $0.84(0.82-0.88)$ & $0.84(0.82-0.88)$ & $0.89(0.85-0.91)^{\mathrm{cfi}}$ \\
\hline WHR of BF & $0.87(0.84-0.90)$ & $0.89(0.86-0.92)$ & $0.88(0.85-0.91)$ & $0.91(0.90-0.94)^{\mathrm{cfi}}$ \\
\hline $\mathrm{TC}(\mathrm{mmol} / \mathrm{L})$ & $4.92 \pm 0.93$ & $4.83 \pm 0.92$ & $5.12 \pm 1.02$ & $5.25 \pm 0.81^{\mathrm{e}}$ \\
\hline $\mathrm{HDL}-\mathrm{C}(\mathrm{mmol} / \mathrm{L})$ & $1.44 \pm 0.24$ & $1.50 \pm 0.30$ & $1.51 \pm 0.34$ & $1.60 \pm 0.44^{b}$ \\
\hline LDL-C (mmol/L) & $2.72 \pm 0.67$ & $2.30 \pm 0.78$ & $2.98 \pm 0.76$ & $2.99 \pm 0.63$ \\
\hline $\mathrm{TG}(\mathrm{mmol} / \mathrm{L})$ & $1.12 \pm 0.57$ & $1.12 \pm 0.44$ & $1.27 \pm 0.64$ & $1.68 \pm 1.45^{\text {be }}$ \\
\hline $\mathrm{FPG}(\mathrm{mmol} / \mathrm{L})$ & $4.92 \pm 0.47$ & $4.99 \pm 0.44$ & $5.10 \pm 0.53$ & $5.39 \pm 0.55^{\mathrm{cfi}}$ \\
\hline
\end{tabular}

Table 2. Comparison of characteristics between different groups in human adjusted for age. Analysis of covariance was carried out to compare the difference between the four groups in human adjusted for age. Log10 transformed FINS, FPG, HOMA-IR, and TG were used for statistical analysis. ARSIN transformed WHR of BF, COS transformed WHR and SQRT transformed BMI were used for statistical analysis. ${ }^{\mathrm{b}} P<0.05$, ${ }^{\mathrm{c}} P<0.01$ vs group C. ${ }^{\text {e}} P<0.05,{ }^{\text {f }} P<0.01$ vs group L. ${ }^{\mathrm{h}} P<0.05,{ }^{\mathrm{i}} \mathrm{P}<0.01$ vs group $\mathrm{M}$.

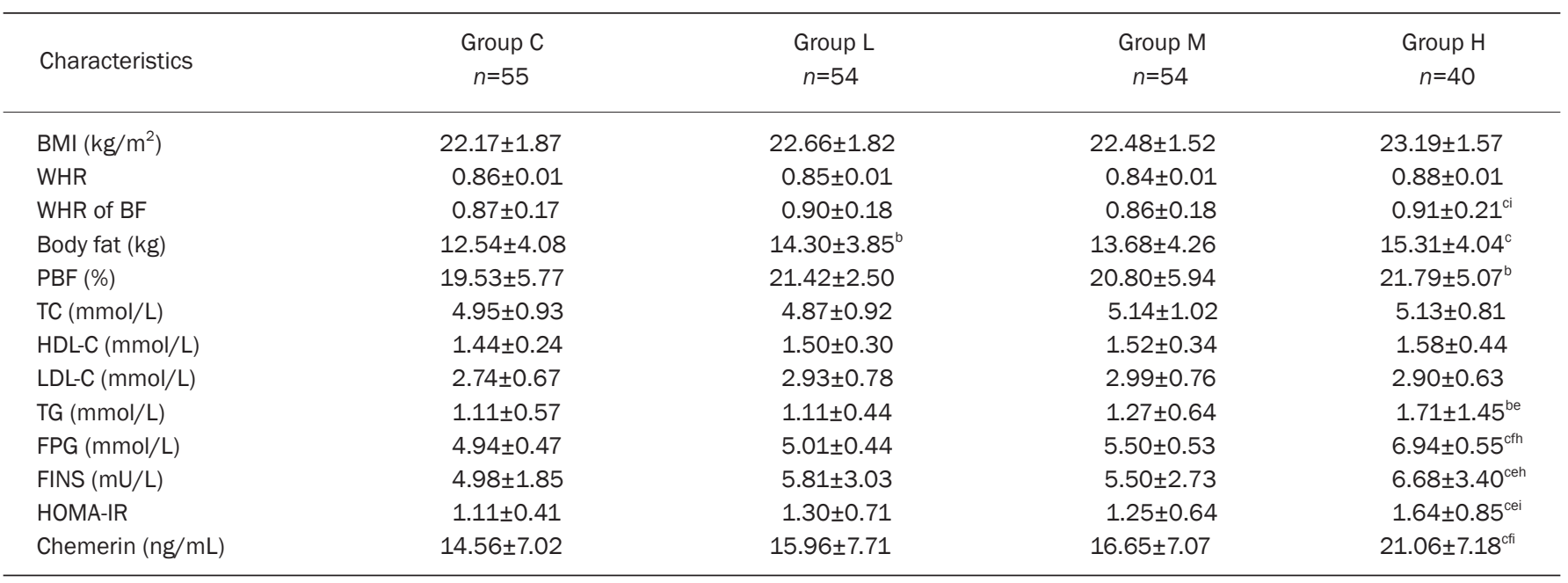

increased significantly compared to the control group after the 22-week treatment (Table 5).

\section{Chronic ethanol treatment increased the chemerin levels in the sera and VAT of the rats}

To observe changes in chemerin of rats after an ethanol treatment, we measured the chemerin levels in the sera, livers, and adipose tissues of rats with and without an ethanol treat- ment. The sera chemerin concentrations of rats in group $\mathrm{M}$ and group $\mathrm{H}$ were significantly higher than those in group $\mathrm{C}$ (18.26 \pm 3.07 and $22.00 \pm 5.96$ vs $14.57 \pm 1.39 \mathrm{pg} / \mathrm{mL}$, respectively; both $P<0.01$ ) (Figure 1A). Similarly, the chemerin levels in the adipose tissues from group $\mathrm{M}$ and group $\mathrm{H}$ were elevated compared to those from group $C(3.87 \pm 0.69$ and $4.31 \pm 1.08$ vs $2.87 \pm 0.74 \mathrm{ng} / 100 \mathrm{mg}$ protein, respectively; $P<0.05$ and $P<0.01$, respectively) (Figure $1 \mathrm{~B})$. The chemerin levels in the livers 
Table 3. Correlation between serum chemerin concentration and metabolic variables.

\begin{tabular}{lcr}
\hline \multicolumn{1}{c}{ Variables } & $r$ & $P$ \\
\hline Age $($ year $)$ & -0.022 & 0.752 \\
BMI $\left(\mathrm{kg} / \mathrm{m}^{2}\right)$ & 0.240 & 0.001 \\
WHR & 0.122 & 0.085 \\
WHR of BF & 0.029 & 0.686 \\
Body fat $(\mathrm{kg})$ & 0.171 & 0.015 \\
PBF $(\%)$ & 0.097 & 0.171 \\
TC $(\mathrm{mmol} / \mathrm{L})$ & 0.014 & 0.846 \\
HDL-C $(\mathrm{mmol} / \mathrm{L})$ & -0.156 & 0.026 \\
LDL-C $(\mathrm{mmol} / \mathrm{L})$ & 0.011 & 0.877 \\
TG $(\mathrm{mmol} / \mathrm{L})$ & 0.339 & $<0.001$ \\
FPG $(\mathrm{mmol} / \mathrm{L})$ & 0.261 & $<0.001$ \\
FINS $(\mathrm{mU} / \mathrm{L})$ & 0.499 & $<0.001$ \\
HOMA-IR & 0.529 & $<0.001$ \\
\hline
\end{tabular}

Table 4. Linear regression analysis of variables associated with serum chemerin levels in human. In multiple linear regression analysis using sera chemerin as dependent varable, values included were TG, FPG, FINS, and HOMA-IR.

\begin{tabular}{lcc}
\hline \multicolumn{1}{c}{ Independent } & $\beta$ & $P$ \\
\hline Age $($ year) & 0.001 & 0.263 \\
BMI $\left(\mathrm{kg} / \mathrm{m}^{2}\right)$ & 0.009 & 0.328 \\
WHR & 0.063 & 0.646 \\
WHR of BF & 0.001 & 0.957 \\
Body fat $(\mathrm{kg})$ & -0.004 & 0.667 \\
PBF $(\%)$ & -0.001 & 0.821 \\
TC $(\mathrm{mmol} / \mathrm{L})$ & -0.041 & 0.230 \\
HDL-C $(\mathrm{mmol} / \mathrm{L})$ & 0.013 & 0.768 \\
LDL-C $(\mathrm{mmol} / \mathrm{L})$ & 0.035 & 0.376 \\
TG $(\mathrm{mmol} / \mathrm{L})$ & 0.056 & 0.001 \\
FPG $(\mathrm{mmol} / \mathrm{L})$ & 0.154 & $<0.001$ \\
FINS $(\mathrm{mU} / \mathrm{L})$ & 0.117 & 0.001 \\
HOMA-IR & 0.387 & 0.011 \\
\hline
\end{tabular}
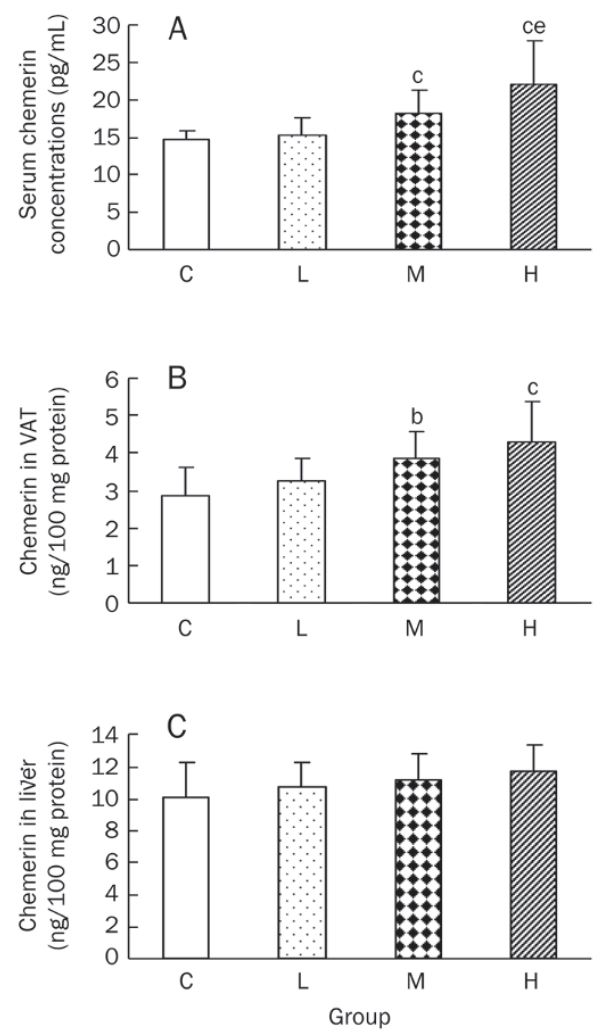

Figure 1. The effects of chronic ethanol treatment on the chemerin levels in serum (A), VAT (B), and liver (C) of rats. Wistar rats in group C, group L, group $\mathrm{M}$, and group $\mathrm{H}$ were fed with edible ethanol doses of $0,0.5,2.5$, and $5.0 \mathrm{~g}^{\mathrm{kg}} \mathrm{k}^{-1} \cdot \mathrm{d}^{-1}$, respectively, for 22 weeks. The chemerin levels in the sera, VATs and livers were measured by ELISAs. The values are given as the mean $\pm S D$ ( $n=9$ in group $C ; n=6$ in group L, group $M$ and group H). VAT, visceral adipose tissue. ${ }^{b} P<0.05,{ }^{c} P<0.01$ vs group $C .{ }^{e} P<0.05$ vs group $L$.

also had a tendency to rise, but the increase was not statistically significant (Figure 1C).

\section{Positive correlation of chemerin levels between the serum and VAT}

To clarify the source of the elevated chemerin levels, we con-

Table 5. Characterization of rats treated with different dosages of ethanol. Wistar rats in group $\mathrm{C}$, group L, group $\mathrm{M}$ and group $\mathrm{H}$ were fed with edible ethanol at doses of $0,0.5,2.5$, and $5.0 \mathrm{~g}^{\mathrm{kg}} \mathrm{kg}^{-1} \cdot \mathrm{d}^{-1}$ for 22 weeks, respectively. Data are mean \pm SD and analyzed by ANOVA. BW, body weight; EAT, ratio of epididymal adipose tissue weight to body weight; PAT, ratio of perinephric adipose tissue weight to body weight; FPG, fasting plasma glucose concentration; FINS, fasting insulin concentration; HOMA-IR, homeostatic model assessment of insulin. ${ }^{\mathrm{b}} P<0.05,{ }^{\mathrm{C}} P<0.01$ vs group C; ${ }^{\mathrm{e}} P<0.05,{ }^{\mathrm{f}} P<0.01$ vs group $L ;{ }^{h} P<0.05$ vs group $M$.

\begin{tabular}{lcccc}
\hline Characteristics & $\begin{array}{c}\text { Group C } \\
n=9\end{array}$ & $\begin{array}{c}\text { Group L } \\
n=6\end{array}$ & $\begin{array}{c}\text { Group M } \\
n=6\end{array}$ & $\begin{array}{c}\text { Group H } \\
n=6\end{array}$ \\
\hline Initia BW (g) & $221.56 \pm 9.13$ & $223.83 \pm 11.57$ & $219.33 \pm 13.03$ & $225.00 \pm 11.44$ \\
Final BW (g) & $436.00 \pm 16.65$ & $433.67 \pm 14.36$ & $413.00 \pm 14.18^{\text {ce }}$ & $408.83 \pm 15.11^{\text {ce }}$ \\
EAT (\% of BW) & $0.93 \pm 0.07$ & $0.92 \pm 0.07$ & $0.98 \pm 0.08$ & $1.10 \pm 0.11^{\text {ch }}$ \\
PAT (\% of BW) & $1.11 \pm 0.10$ & $1.04 \pm 0.08$ & $1.09 \pm 0.09$ & $3.52 \pm 0.56$ \\
FPG (mmol/L) & $3.72 \pm 0.54$ & $3.45 \pm 0.34$ & $26.31 \pm 12.12$ & $3.55 \pm 0.48$ \\
FINS (mIU/L) & $22.03 \pm 4.96$ & $24.33 \pm 10.34$ & $4.05 \pm 1.71$ & $5.20 \pm 1.91^{\mathrm{b}}$ \\
HOMA-IR & $3.61 \pm 0.83$ & $3.83 \pm 2.08$ & & \\
\hline
\end{tabular}


ducted a correlation analysis between the serum and VAT or liver chemerin (Figure 2). The results showed that the serum chemerin concentrations were positively associated with the chemerin in the VAT after adjusting for the liver chemerin. The relationship between the serum and liver chemerin was not statistically significant after adjusting for the chemerin in the VAT.
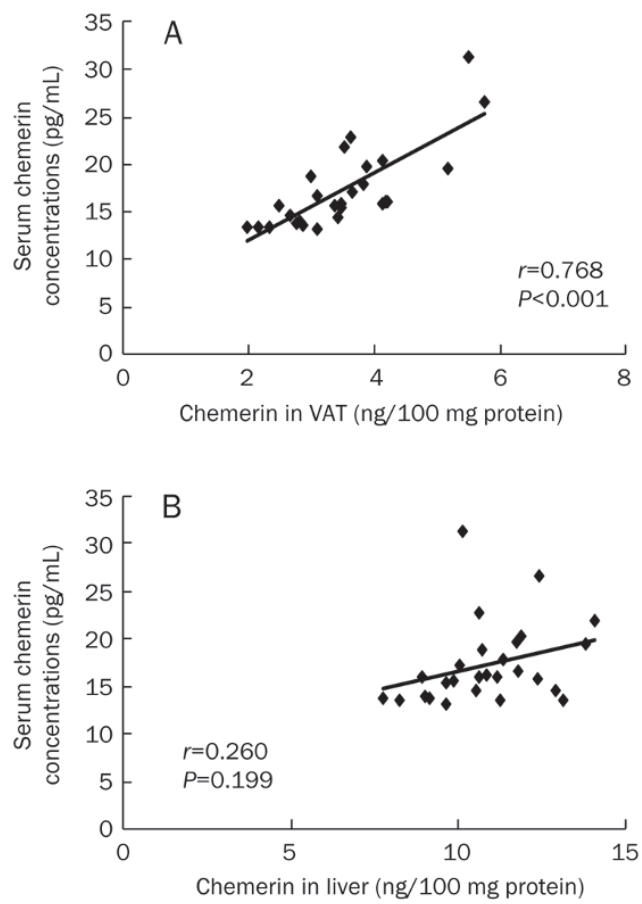

Figure 2. Correlation of the chemerin levels between the sera and tissues of the rats. Wistar rats in group $\mathrm{C}$, group $\mathrm{L}$, group $\mathrm{M}$, and group $\mathrm{H}$ were fed with edible ethanol doses of $0,0.5,2.5$, and $5.0 \mathrm{~g}^{\mathrm{kg}} \mathrm{k}^{-1} \cdot \mathrm{d}^{-1}$, respectively, for 22 weeks. The chemerin levels in the sera, VATs and livers were measured by ELISAs. (A) The correlation between the serum and VAT chemerin levels after adjusting for the liver chemerin. (B) The correlation between the serum and liver chemerin levels after adjusting for the VAT chemerin. VAT, visceral adipose tissue.

\section{Discussion}

In this study, we found that the serum chemerin concentrations in both humans and rats were elevated with increasing doses of ethanol. Furthermore, the serum chemerin was associated with TG and the HOMA-IR in humans. The increase in serum chemerin was mainly attributed to the elevation of chemerin in the VAT of rats.

We observed that ethanol increased the serum chemerin, which was associated with TG in humans. Ethanol may increase chemerin via the mechanisms discussed below. Studies have demonstrated that ethanol increased the circulating free fatty acid (FFA) levels ${ }^{[15,16]}$, which induced chemerin significantly in differentiated 3T3-L1 cells and elevated the soluble chemerin in the supernatant ${ }^{[17]}$. Chronic ethanol ingestion increased the circulating levels of lipopolysaccharide (LPS), and the differentiation of 3T3-L1 cells in the presence of LPS significantly induced cellular and soluble chemerin ${ }^{[17,18]}$. Moreover, our previous study observed that chronic ethanol consumption caused a dose-dependent increase of TNF-a in the sera of rats ${ }^{[11]}$. An in vitro chronic ethanol treatment also increased TNF- $\alpha$ in peripheral blood monocytes ${ }^{[19]}$. TNF-a induced the chemerin mRNA expression in 3T3-L1 adipocytes in a doseand time-dependent manner and increased the bioactive chemerin levels in the mouse serum and primary adipocyte media $^{[8,20]}$. Similarly, chronic alcohol consumption increased interleukin-1-beta (IL-1 $\beta$ ) in vivo and in vitro. In 3T3-L1 adipocytes and brown fat cells, IL-1 $\beta$ stimulated the chemerin gene expression and induced the chemerin protein secretion in a dose-dependent fashion ${ }^{[7,21-24]}$. In addition, we also observed that ethanol increased insulin and the HOMA-IR, which were independently associated with chemerin, in the human study. Tan et al reported that insulin elevated the chemerin level in human adipose tissue explants in vitro and in healthy individuals ${ }^{[25]}$. Conversely, the ethanol-induced increase of chemerin may be involved in ethanol-induced insulin resistance. Previous research demonstrated that chemerin played roles in insulin resistance by decreasing glucose transport and interfering with insulin signaling transduction ${ }^{[6-8]}$. The elevated insulin level that is induced by ethanol led to an increase of chemerin, and the increased chemerin may participate in insulin resistance, thereby forming a vicious cycle.

Although the chemerin levels increased with higher doses of ethanol in the sera of humans and rats, the elevations in the middle-dose group of rats were more significant than those in the humans. Species differences and the ethanol consumption pattern may partly contribute to this phenomenon. The rats were given a fixed dose of ethanol once a day at the same time by a gastric tube over a short period of time, and most of the ethanol was absorbed. In contrast, the human subjects usually drank slowly during meals, and the absorption of ethanol was diminished. Therefore, the peak ethanol concentration in rats was higher than that in humans at the same dose of ethanol, which resulted in a more severe insulin resistance in the rats. Accordingly, the chemerin levels that were induced by the elevated insulin in the rats were higher than those in the humans.

The source of the circulating chemerin is presently unclear. Both the liver and white adipose tissue highly expressed chemerin $^{[3,4]}$. To determine the source of the elevated serum chemerin after the ethanol treatment, we tested the chemerin concentrations in the VAT and liver. The results showed that the chemerin levels in the liver were higher than those in the VAT, but the chemerin levels in the VAT positively correlated with the serum chemerin after adjusting for the liver chemerin. Therefore, we inferred that the increased serum chemerin was mainly attributed to the elevated VAT chemerin after the ethanol treatment. Ethanol significantly increased the VAT in both humans and rats, and the increased adipose tissue may directly secrete more chemerin. Alternatively, ethanol may induce the VAT chemerin via the various mechanisms that were discussed prior. Nevertheless, the consistency of the elevated chemerin in the serum and adipose tissues cannot be 
completely explained. Further research should be conducted to determine the mechanism.

Our study showed that after adjusting for age, the elevation of TG in group $\mathrm{H}$ remained significant. This may be because ethanol increased the FFAs, and more FFAs participated in the anabolism of TG, which led to the increased TG. Whether the ethanol-induced increase of chemerin plays a role in lipid metabolism is unknown, and further research is required for elucidation. Our study also found that the WHR and waisthip ratio of body fat in the high-dose group were significantly higher than those in the control group. Similarly, the relative weights of the epididymal adipose tissue increased in the highdose group of rats even though their body weight decreased. In this study, we observed that the high-dose ethanol increased insulin and the HOMA-IR in both humans and rats. Many studies have demonstrated that insulin was responsible for primary preadipocyte differentiation and confirmed its adipogenic effect $t^{[26-28]}$. Elevated insulin, which was induced by ethanol, may increase the fat mass by its adipogenic effect. Alternatively, an ethanol treatment also upregulated sterol regulatory element-binding protein-1 (SREBP-1), and SREBP-1 activated a cascade of genes that are required for endogenous lipogenesis and pre-adipocyte differentiation ${ }^{[29,30]}$. In addition, the ethanol-induced increase of chemerin may also participate in adipocyte differentiation, thereby leading to the elevation of fat mass ${ }^{[3,4]}$. Meanwhile, the increased fat might synthesize and secrete more chemerin, which is involved in adipocyte differentiation, and thereby generate a vicious cycle of elevated adipogenesis.

This study had some limitations. First, because there are fewer female drinkers than male drinkers and to eliminate the effects of endogenous sex hormones, we chose to study only male drinkers. Accordingly, we selected male Wistar rats for the animal experiment. Second, samples of adipose tissue and livers from humans were difficult to acquire, and therefore, we selected rats for the study of chemerin levels in tissues. In addition, although the current study was capable of identifying correlations between ethanol and chemerin, further experimental studies are required to unravel the molecular mechanism for the observed associations between ethanol and chemerin.

In conclusion, our study demonstrates that ethanol treatment increases serum chemerin and that chemerin levels are associated with several key parameters of metabolic syndrome. Moreover, the ethanol-induced elevation of serum chemerin was mainly derived from adipose tissue. Chemerin may participate in chronic, ethanol-induced insulin resistance and lipid metabolism disorder. Lower TG and reduced body fat may decrease the chemerin level and consequently decrease the insulin resistance that is induced by ethanol.

\section{Acknowledgements}

We thank the staff of the central laboratory at the Provincial Hospital that is affiliated with Shandong University for their pertinent advice and excellent technical assistance.

This work was supported by the National Natural Science
Foundation of China (№ 30940038) and the Natural Science Foundation of Shandong Province, China (№ ZR2009CM008).

\section{Author contribution}

Jia-jun ZHAO and Qing-bo GUAN designed the research; Ruizhen REN was responsible for the practical parts of the study, including participating in the epidemiological investigation, conducting experiments, analyzing the data and writing the article; Xu ZHANG participated in the epidemiological investigation and the experiments; Jin XU, Hai-qing ZHANG, and Ming-feng $\mathrm{CAO}$ participated in the epidemiological investigation and human sera collection; Chun-xiao YU and Ling GAO supervised the experiments; and Ling GAO was also responsible for revising the manuscript.

\section{References}

1 Rosen ED, Spiegelman BM. Adipocytes as regulators of energy balance and glucose homeostasis. Nature 2006; 444: 847-53.

2 Nagpal S, Patel S, Jacobe H, DiSepio D, Ghosn C, Malhotra M, et al. Tazarotene-induced gene 2 (TIG2), a novel retinoid-responsive gene in skin. J Invest Dermatol 1997; 109: 91-5.

3 Goralski KB, McCarthy TC, Hanniman EA, Zabel BA, Butcher EC, Parlee $\mathrm{SD}$, et al. Chemerin, a novel adipokine that regulates adipogenesis and adipocyte metabolism. J Biol Chem 2007; 282: 28175-88.

4 Roh SG, Song SH, Choi KC, Katoh K, Wittamer V, Parmentier M, et al. Chemerin - a new adipokine that modulates adipogenesis via its own receptor. Biochem Biophys Res Commun 2007; 362: 1013-8.

5 Wittamer V, Franssen JD, Vulcano M, Mirjolet JF, Le Poul E, Migeotte I, et al. Specific recruitment of antigen-presenting cell by chemerin, a novel processed ligand from human inflammatory fluids. J Exp Med 2003; 198: 977-85.

6 Becker M, Rabe K, Lebherz C, Zugwurst J, Göke B, Parhofer KG, et al. Expression of human chemerin induces insulin resistance in the skeletal muscle but does not affect weight, lipid levels and atherosclerosis in LDL receptor knockout mice on high fat diet. Diabetes 2010; 59: 2898-903.

7 Kralisch S, Weise S, Sommer G, Lipfert J, Lossner U, Bluher M, et al. Interleukin-1 beta induces the novel adipokine chemerin in adipocytes in vitro. Regul Pept 2009; 154: 102-6.

8 Sell H, Laurencikiene J, Taube A, Eckardt K, Cramer A, Horrighs A, et al. Chemerin is a novel adipocyte-derived factor inducing insulin resistance in primary human skeletal muscle cells. Diabetes 2009; 58: $2731-40$.

9 Greenfield JR, Samaras K, Jenkins AB, Kelly PJ, Spector TD, Campbell LV. Moderate alcohol consumption, estrogen replacement therapy and physical activity are associated with increased insulin sensitivity: is abdominal adiposity the mediator? Diabetes Care 2003; 26 : 2734-40.

10 Kang L, Sebastian BM, Pritchard MT, Pratt BT, Previs SF, Nagy LE. Chronic ethanol-induced insulin resistance is associated with macrophage infiltration into adipose tissue and altered expression of adipocytokines. Alcohol Clin Exp Res 2007; 31: 1581-8.

11 Yu HC, Li SY, Cao MF, Jiang XY, Feng L, Zhao JJ, et al. Effects of chronic ethanol consumption on levels of adipokines in visceral adipose tissues and sera of rats. Acta Pharmacol Sin 2010; 31: 4619.

12 Koppes LL, Dekker JM, Hendriks HF, Bouter LM, Heine RJ. Moderate alcohol consumption lowers the risk of type 2 diabetes: a metaanalysis of prospective observational studies. Diabetes Care 2005; 28: $719-25$. 
13 Yoon YS, Oh SW, Baik HW, Park HS, Kim WY. Alcohol consumption and the metabolic syndrome in Korean adults: the 1998 Korean National Health and Nutrition Examination Survey. Am J Clin Nutr 2004; 80: 217-24.

14 Matthews DR, Hosker JP, Rudenski AS, Naylor BA, Treacher DF, Turner RC. Homeostasis model assessment: insulin resistance and beta-cell function from fasting plasma glucose and insulin concentrations in man. Diabetologia 1985; 28: 412-9.

15 Nanji AA, Dannenberg AJ, Jokelainen K, Bass NM. Alcoholic liver injury in the rat is associated with reduced expression of peroxisome proliferator-alpha (PPARalpha)-regulated genes and is ameliorated by PPARalpha activation. J Pharmacol Exp Ther 2004; 310: 417-24.

16 You M, Fischer M, Deeg MA, Crabb DW. Ethanol induces fatty acid synthesis pathways by activation of sterol regulatory element-binding protein (SREBP). J Biol Chem 2002; 277: 29342-7.

17 Bauer S, Wanninger J, Schmidhofer S, Weigert J, Neumeier M, Dorn C, et al. Sterol regulatory element-binding protein 2 (SREBP2) activation after excess triglyceride storage induces chemerin in hypertrophic adipocytes. Endocrinology 2011; 152: 26-35.

18 Fukui H, Brauner B, Bode J, Bode C. Plasma endotoxin concentrations in patients with alcoholic and non-alcoholic liver disease: reevaluation with an improved chromogenic assay. J Hepatol 1991; 12: 162-9.

19 Kishore R, Hill JR, McMullen MR, Frenkel J, Nagy LE. ERK1/2 and Egr-1 contribute to increased TNF-alpha production in rat Kupffer cells after chronic ethanol feeding. Am J Physiol Gastrointest Liver Physiol 2002; 282: G6-15.

20 Parlee SD, Ernst MC, Muruganandan S, Sinal CJ, Goralski KB. Serum chemerin levels vary with time of day and are modified by obesity and tumor necrosis factor-\{alpha\}. Endocrinology 2010; 151: 2590-602.

21 Laso FJ, Vaquero JM, Almeida J, Marcos M, Orfao A. Production of inflammatory cytokines by peripheral blood monocytes in chronic alcoholism: relationship with ethanol intake and liver disease. Cytometry B Clin Cytom 2007; 72: 408-15.

22 Ahluwalia B, Wesley B, Adeyiga O, Smith DM, Da-Silva A, Rajguru S. Alcohol modulates cytokine secretion and synthesis in human fetus: an in vivo and in vitro study. Alcohol 2000; 21: 207-13.

23 Valles SL, Blanco AM, Azorin I, Guasch R, Pascual M, Gomez-Lechon MJ, et al. Chronic ethanol consumption enhances interleukin-1mediated signal transduction in rat liver and in cultured hepatocytes. Alcohol Clin Exp Res 2003; 27: 1979-86.

24 Hsiang CY, Wu SL, Cheng SE, Ho TY. Acetaldehyde-induced interleukin-1 beta and tumor necrosis factor-alpha production is inhibited by berberine through nuclear factor-kappaB signaling pathway in HepG2 cells. J Biomed Sci 2005; 12: 791-801.

25 Tan BK, Chen J, Farhatullah S, Adya R, Kaur J, Heutling D, et al. Insulin and metformin regulate circulating and adipose tissue chemerin. Diabetes 2009; 58: 1971-7.

26 Geloen A, Collet AJ, Guay G, Bukowiecki LJ. Insulin stimulates in vivo cell proliferation in white adipose tissue. Am J Physiol 1989; 256: c190-6.

27 Suryawan A, Swanson LV, Hu CY. Insulin and hydrocortisone, but not triiodothyronine, are required for the differentiation of pig preadipocytes in primary culture. J Anim Sci 1997; 75: 105-11.

28 Deslex S, Negrel R, Ailhaud G. Development of a chemically defined serum-free medium for differentiation of rat adipose precursor cells. Exp Cell Res 1987; 168: 15-30.

29 You M, Fischer M, Deeg MA, Crabb DW. Ethanol induces fatty acid synthesis pathways by activation of sterol regulatory element-binding protein (SREBP). J Biol Chem 2002; 277: 29342-7.

$30 \mathrm{Kim} \mathrm{JB}$, Spiegelman BM. ADD1/SREBP1 promotes adipocyte differentiation and gene expression linked to fatty acid metabolism. Genes Dev 1996; 10: 1096-107. 